\title{
DUPUYTREN'S CONTRACTURE
}

By J. S. R. Golding, F.R.C.S.

Senior Registrar, Department of Plastic Surgery, The Middlesex Hospital

Astley Cooper recognized a contracture of the palmar fascia in the hand and described its treatment by subcutaneous fasciotomy (1822), but it was Dupuytren (1834) who clearly differentiated, from a mass of other conditions causing clawing of the fingers, the peculiar condition to which his name has been given.

The contracture usually starts as a slightly tender nodúle, later becoming hard and painless, in the palm proximal to the ring finger. The skin becomes attached to the nodule and a small, pitlike 'pucker' develops. The thickening gradually progresses and nodules appear in the finger itself, which becomes progressively more flexed at the metacarpo-phalangeal and proximal interphalangeal joints, and extended at the distal joint.

Since the time of Astley Cooper many attempts have been made to devise a satisfactory treatment for the condition, but with so little success that significant published results have been extremely few until recent years, when a firmer grasp of the pathology and anatomy of the condition has enabled a satisfactory technique of treatment to be evolved, giving excellent results in over 80 per cent. of cases (Skoog, 1948).

\section{Anatomy}

The palmar fascia is morphologically the termination of the palmaris longus muscle, which is primitively the flexor of the metacarpo-phalangeal joints and is present in about 90 per cent. of people. It is noteworthy, however, that Dupuytren's contracture can occur in the absence of a palmaris longus.

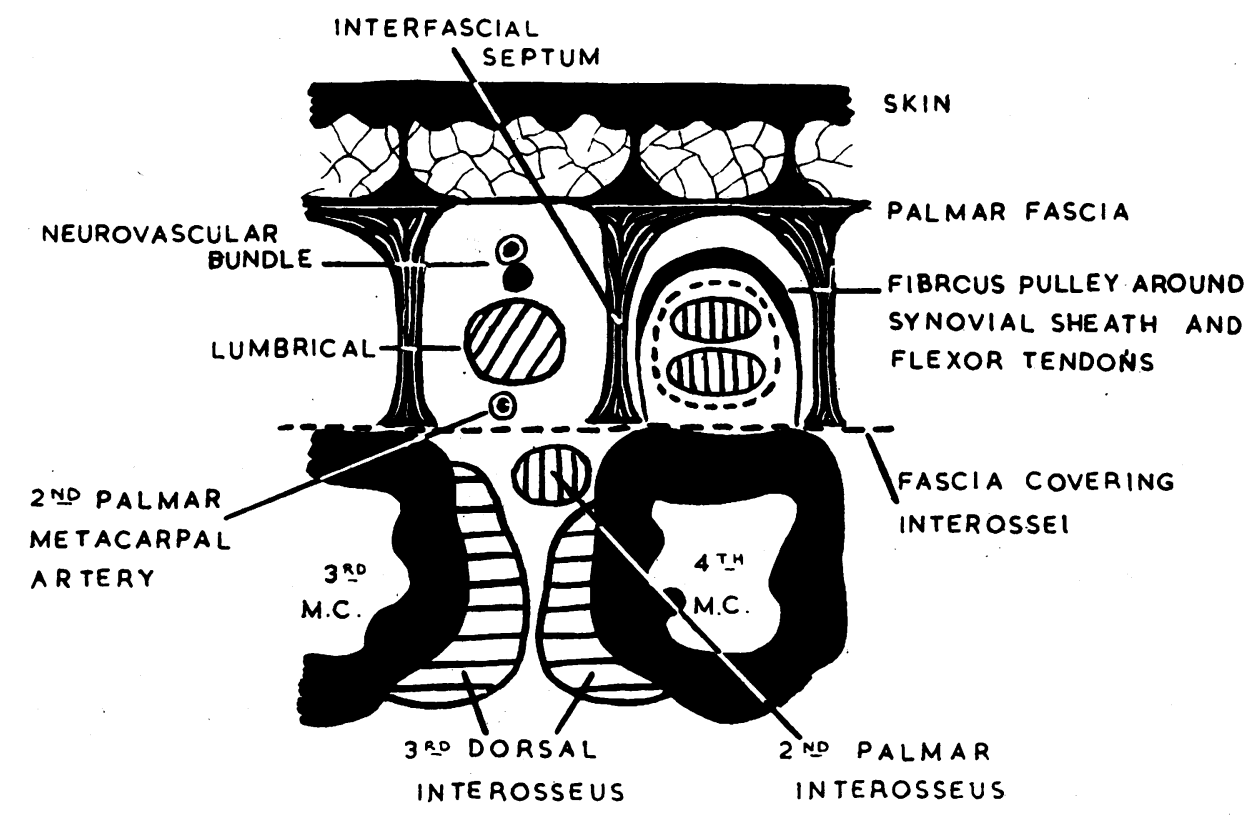

DIAGRAM TO SHOW THE PALMAR FASCIA AND INTERFASCIAL SEPTA

JUST PROXIMAL TO THE HEAD OF THE GTHMETACARPAL BONE.

FIG. 1.-Diagram to show the palmar fascia and the interfascial septa immediately proximal to the head of the fourth metacarpal bone. 


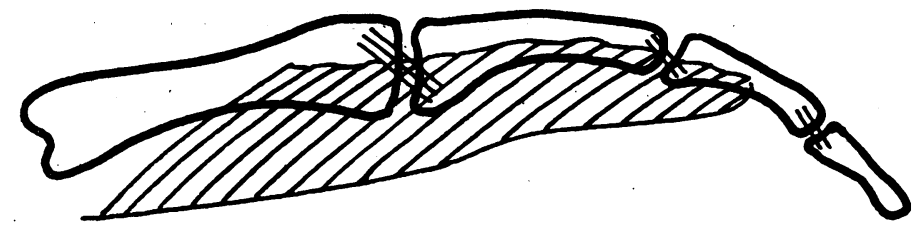

FIG. 2.-Diagram of a metacarpal bone and phalanges, to show the attachment of the palmar fascial slips to the sides of the phalanges.

The palmar fascia binds the palmar skin to the metacarpal bones. It adheres to the skin intimately but is not attached to the neurovascular bundles or to the tendon sheaths. It is thicker on the ulnar than on the radial side of the hand and is continuous at its margins with the fascia over the thenar and hypothenar eminences. In the distal third of the palm, longitudinal septa pass down on either side of the tendons to be attached, with the fascia over the interossei, to the sides of the metacarpal bones. Within these tunnels run the tendons which are separated by the septa from the neurovascular bundles and lumbrical muscles. These septa are attached to the transverse metacarpal ligament and capsule of the joints. There is also a thin fibrous sheath covering the tendons which, in the region of the heads of the metacarpal bones, is thickened to form a 'pulley' preventing the tendons from 'bow-stringing' across the metacarpo-phalangeal joints. This fibrous sheath is distinct from and is not affected by the contraction of the fascia (Fig. I).

Distally the fascia divides into a strip for each finger, which passes down to be attached to the sides of the proximal phalanx and to the proximal half of the middle phalanx (Fig. 2).

The digital nerve to the fifth finger may be anomalous and may divide to the radial side of its web, to run obliquely behind the palmar fascia to reach the ulnar side of the finger. The nerve passes obliquely over the tendon sheath and behind the palmar fascia in any position between the distal palmar crease and the web. If it is not first identified in the neurovascular bundle proximally and followed distally, it is liable to injury.

\section{Aetiology}

The condition is more than eight tirnes as common in males as in females. It usually appears between the ages of 30 and $4 \circ$ years and progresses at a varying rate. In more than half the cases it is bilateral. In unilateral cases it is twice as common in the right hand as in the left. Very frequently a history of familial incidence can be elicited.

The fourth and fifth fingers are those usually affected. Skoog (1948) reports that in 64 per cent. of cases the fourth finger and in 53 per cent. of cases the fifth finger is affected. The third finger is affected in 24 per cent. and the index finger in 5 per cent. of cases. The thumb is affected in only about 3 per cent. of cases.

There is no conclusive evidence of any association of Dupuytren's contracture with any general disease except epilepsy, or with any particular occupation, although Niederland (1932) reported the high incidence of 17.8 per cent. in certain textile workers using the palms of their hands in a particularly vigorous manner. However, there is some evidence that the severity of the contracture is greater where heavy manual work has been done. Cases with a strong hereditary factor tend to have several fingers affected (Kanavel, et al., r929).

There is no doubt that epileptics are particularly affected. Lund (I94I) found that about 50 perr cent. of 190 male epileptics and 25 per cent. of female epileptics showed the condition. He remarks that earlier workers did not notice this association, and in fact Feré (quoted by Lund) writing early this century categorically stated that Dupuytren's contracture was rare in epileptics. Lund suggests that the advent of the barbiturates in the treatment of epilepsy may be the true cause of this remarkable association.

The histological changes associated with Dupuytren's contracture are also found on the backs of the hands, where they are known as knuckle pads, and in the soles of the feet.

\section{Knuckle Pads (Fig. 3b)}

These were first described by Paget (1875) in association with gout. Garrod (1893) noticed the association with Dupuytren's contracture. The pads are situated over the proximal interphalangeal joints and are commonly attached to the overlying skin (Fig. 3). Microscopically the changes are identical with those seen in the palm. Lund (194r) noticed knuckle pads in 18 per cent. of epileptics without Dupuytren's contracture and in 38 per cent. of epileptics with the condition.

Dupuytren's Contracture Affecting the Plantar Fascia

This condition is rare. Bunnell (1944) noticed 


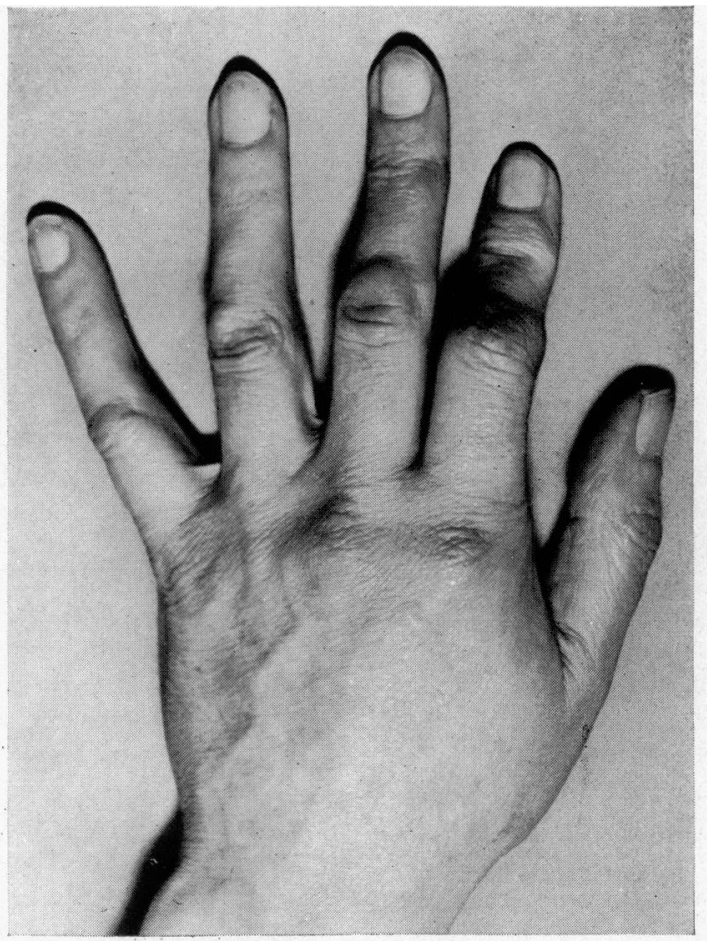

(a)

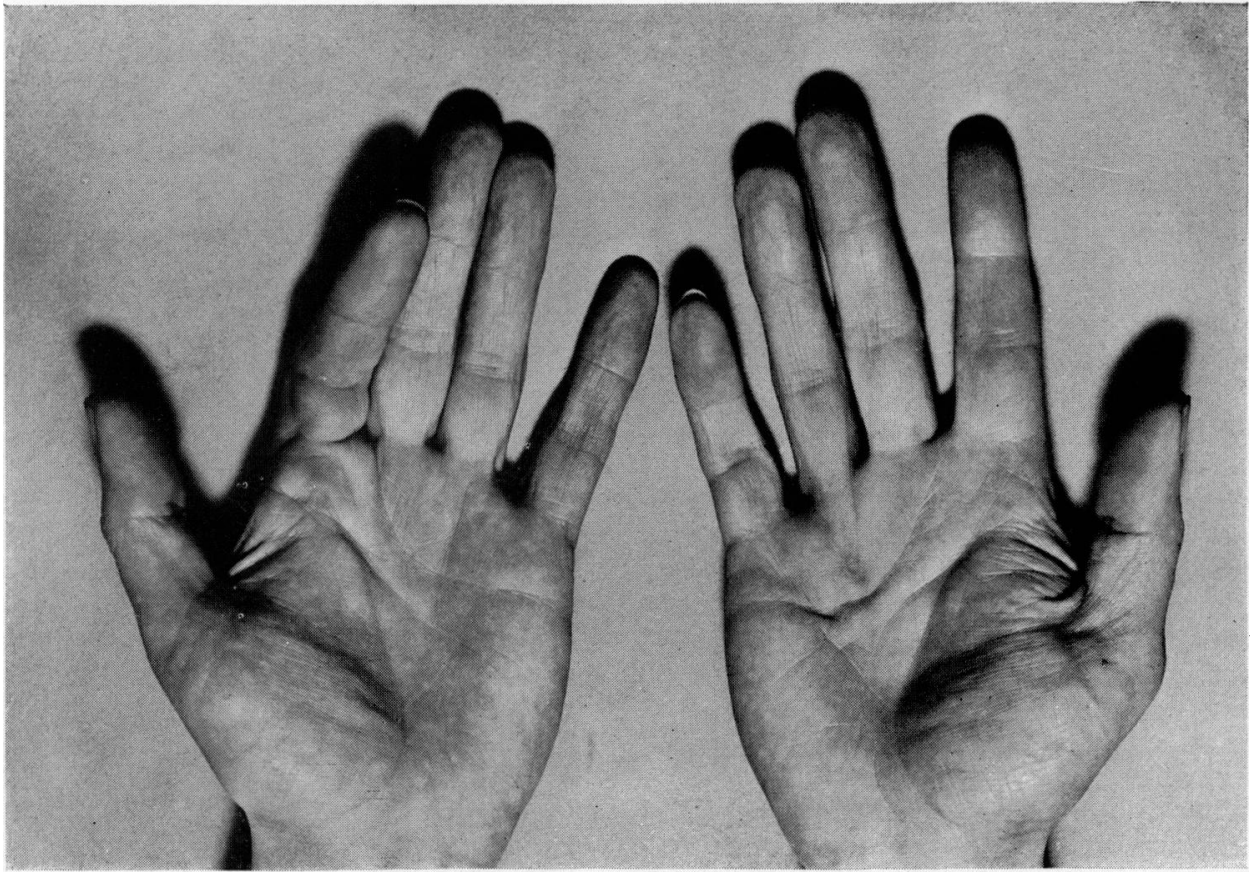

(b)

Fig. 3.-Photographs showing the distribution of afiected fascia in a man of 35 years with a strong family history of Dupuytren's contracture (b). Knuckle pads are well marked in this case (a). 
it in three of the 87 hand cases he had operated on. Skoog (1948) reported a case where the feet were affected before the hands. Considering the considerable trauma to which the soles of the feet are subjected, it would be expected far more frequently if the condition had a purely traumatic aetiology.

\section{Pathology}

The excised fascia shows a dense mass of hyperplastic and irregular fibrous connective tissue. In this there are hypercellular areas which interrupt the fibres rather than displace them. Iron pigment may be found.

Meyerding (I94I) believed the process to be a chronic inflammatory reaction beginning in the interstitial fibrous tissue of the palm. Horwitz (1942), comparing the tissue with that found in normal hands at autopsy, concluded that the process was a 'benign fibroplasia' and stressed the great similarity with the histology of keloid scars and fascial desmoids. However, patients with Dupuytren's contracture show no tendency to form either keloids or desmoids.

Skoog (1948) characterizes the condition by:-

I. All structures in the palm are normal and distinct from the contracted fascia.

2. Nerves may be displaced but are never infiltrated.

3. The subcutaneous tissue is displaced because of its attachment to the contracting cords of fibrous tissue.

Skoog believes that the contracture is the result of multiple partial ruptures occurring in a degenerating palmar aponeurosis which heals by scar formation. The iron pigment is the result of minute haemorrhages, and the hypercellular regions are recent ruptures in the process of repair. This accounts for the interruption of the fibres.

\section{Treatment}

Once this progressive fibrosis has commenced we know that it will continue. Nothing less than excision of the whole affected tissue will give lasting relief. Operation deferred until capsular changes have developed in the interphalangeal joints cannot give perfect results. Where both hands are involved that less affected should be treated first, to lessen the incapacity of the patient. The many methods, such as subcutaneous fasciotomy, which have been used in the past have been entirely discarded except in certain cases as an adjunct to excision because recurrence is inevitable.

Recently vitamin ' $\mathrm{E}$ ' has been tried (Steinberg, 1947) in the early active stage of the disease, but the results have been unsatisfactory (King,
1949). The only effective treatment is complete excision of the palmar fascia and its septa.

\section{Operative Technique}

It is essential to perform this delicate procedure in an avascular field. An Esmarch bandage is applied to the upper arm and above this a bloodpressure cuff is inflated to a pressure of $250 \mathrm{~mm}$. Hg. The bandage is then removed. It $\stackrel{\mathbb{Q}}{2}$ is safe to maintain this pressure for an hour. The cuff is then deflated for 10 minutes whilst a swab soaked in saline is pressed into the wound (Bruner, 195I). This procedure should be repeated at half-hourly intervals.

The hand is prepared with cetavlon and spirit; no coloured antiseptic solution should be used or difficulty will be encountered in ensuring that the blood flow is sufficient when the pressure dressing has been applied after operation.

Normally complete excision of the fascia can be obtained through a transverse incision in the distal skin crease of the palm, together with a ' $Z$ ' incision over the proximal phalanx of the finger (Fig. 4). In cases where the palmar skin is short and severely affected the ' $\mathrm{Z}$ ' palmar incision is used and the flaps later transposed to give additional length. Rarely a small split skin grafe may be needed to give the skin cover which io essential if the danger of sepsis is to be averted.

Preliminary subcutaneous fasciotomy has bee advised by Bunnell (1944), where such severe flexion of the fingers is present as to prevent exposure of the palm. A cataract knife is used to divide the main cords subcutaneously and a splint or rubber sponge is bandaged to the palm for to to I 4 days to lengthen the skin.

The palmar incision is made and the skin carefully dissected up from the fascia. The skin edges are raised with skin hooks to prevent subsequent necrosis. If access is found to be difficult owing to flexion of the fingers, the thickened cord-like areas are divided transversely to allow the fingers to extend before the flaps are dissected free. The palmar fascia is then detached proximally. If there is difficulty in exposing the proximal part of the fascia, a small curved incision is made over the flexor retinaculum (Fig. 4). Particular attention is paid to the removal of the septa on either side of the tendons by retracting the neurovascular burdles and lumbrical muscles to expose the metacarpal bones. These septa are divided close to the metacarpal bones. Great care is necessary to avoid damage to the digital nerves, which are followed distally into the base of each affected finger.

When the whole fascia has been dissected free, attention is turned to the fingers. The palmar surface of the proximal phalanx is incised as a 


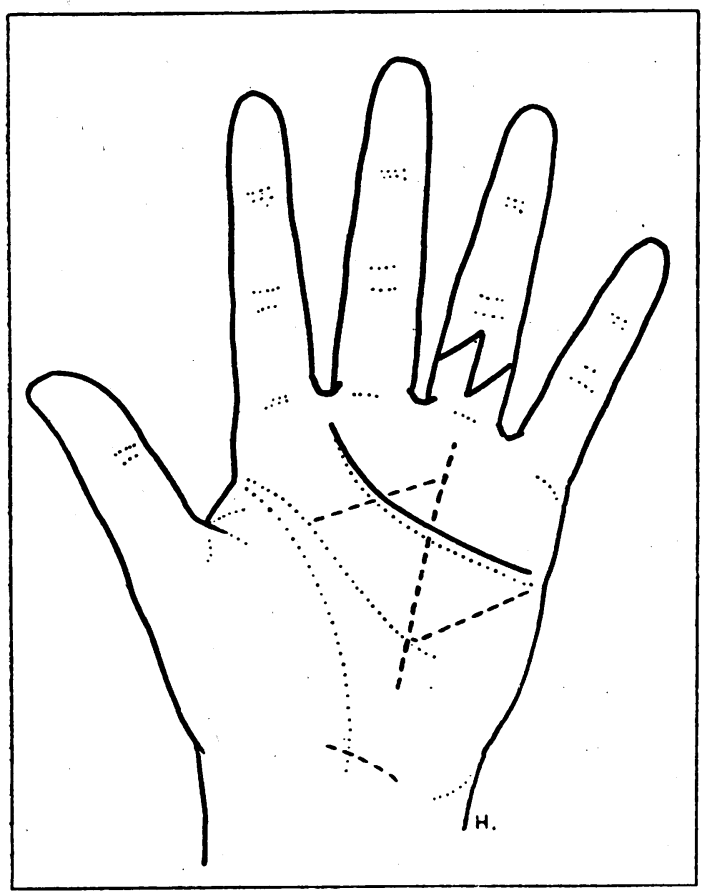

FIG. 4--A diagram showing the incisions commonly used. The central part of the fascia is exposed through an incision parallel to the distal palmar crease. The digital extensions are excised through incisions on the fingers, as shown, whilst the fascia is separated proximally from the flexor retinaculum through a small curved incision if this is necessary.

' $Z$ ', and the flaps dissected free. The block of fascia previously dissected is divided into a portion for each affected finger and the bridge of skin between the palm and finger undermined to allow the fascia to be passed through. This exposes the digital neuro-vascular bundles which are then followed up from the palm into the finger and the fascia is dissected away from them and detached from the sides of the phalanx. The fascia is followed up until normal tissue of the middle phalanx is reached and then divided.

The two flaps of the finger are transposed, utilizing the transverse width of skin to give the additional length which is usually needed. The flaps are carefully sutured, to avoid damage to the skin edges. Toothed forceps should not be used. After operation a crepe bandage is applied firmly over about an inch of cotton wool to give even pressure. Cotton wool is placed between each finger, which makes the hand much more comfortable. The blood pressure cuff is then released and removed and the fingers inspected to ensure that the pressure bandage has not been applied too tightly.

For the first 24 hours the hand is suspended to diminish swelling. The pressure dressing is left in place for 10 days; during this time the cotton wool between the fingers is removed if it is preventing the finger movements which are started on the third post-operative day. Half the stitches are removed on the tenth day, and the remainder retained until the fourteenth day because the palm heals slowly.

An intensive course of physiotherapy, including particularly wax baths and active exercises, is commenced as soon as the palm is soundly healed. Usually the finger extension is full after four weeks' treatment.

\section{Results}

In a series of 55 hands operated on in this hospital during the last five years it is too early to gaino exact information about the success of the variouso treatments used. However, in over 75 per cent of cases the results were considered to be good, and the patients have not reported with recurrence. In five cases where fasciotomy was performed, two were later referred for excision and one was reported as being satisfactory; the other two were not satisfactory but refused further surgery. Two cases treated by radiotherapy were unimproved, as were seven cases treated with vitamin $\mathrm{E}$.

The only failure in the group treated by excision was due to an exacerbation of rheumatoid arthritis which had been quiescent for many years. The ten cases giving only fair results were due to delayed healing interfering with early movements in four cases and severe capsular changes limiting extension in the remaining six cases.

My thanks are due to Mr. Rainsford Mowlem, whose technique of operation has been described, for his advice and criticism.

\section{BIBLIOGRAPHY}

BRUNER, J. M. (1931), 尹. Bone ft. Surg., 33, 22 r.

BUNNELL, S. (1944), ' Surgery of the Hand,' Philadelphia.

COOPER, A. (1822), 'Treatise on Dislocations and Fractures of the Joints,' London, p. 524.

DUPUYTREN, G. (1834), Lancet, ii, 222.

GARROD, A. E. (1893), St. Barts. Hosp. Rep., 29, 157.

HORWITZ, T. (1942), Arch. Surg., 44, 687.

KANJ] VI:T, A B. KOCH, S. L., and MASON, M. L. (1929), Surg. Gyn. Ob:t., 48, 145.
KING, R. A. (1949), f. Bone ft. Surg., 31, 443.

LUND, M. (1941), Acta. Psychiat. Neurol., 16, 465.

MEYERDING, H. W. BL.ACK, J. R., and BRODERS, A. C. (1944), Surg. Gyn. Obst., 72, 582. NIEDERLAND, W. (1933), Zbl. Chir., 60, 985. -

SKOOG, T. (1948), Acta. Chir. Scand., 46, Supplement 139. STEINBERG C. (1947), N Y St. F. Med. 47, 1679. 